\title{
Tumor angiogenesis genotyping and efficacy of first-line chemotherapy in metastatic gastric cancer
} patients

\begin{abstract}
Aim: Besides correlating with prognosis, tumor-driven angiogenesis also seemed able to influence response/resistance to chemotherapy in preclinical models. We examined the role of tumor angiogenesis genotyping in determining clinical outcome in metastatic gastric cancer patients receiving first-line chemotherapy. Patients \& methods: VEGF-A, VEGF-C, FLT1, KDR and FLT4 genotyping was analyzed in gastric tumors from patients receiving platinum-based first-line chemotherapy. Results: VEGF-A rs 25648 correlated with response rate (partial response: $18 \%$ among patients showing the VEGF-A rs 25648 CT or TT genotype vs $44 \%$ among patients showing the VEGF-A rs $25648 \mathrm{C}$ genotype; $p=0.04)$. At multivariate analysis only VEGF-A rs 25648 maintained an independent role in determining median progression-free survival (hazard ratio: $1.6595 \% \mathrm{Cl}: 1.12-2.78$ ) and overall survival (hazard ratio: $1.58,95 \% \mathrm{Cl}: 1.17-2.65$ ). Conclusion: VEGF-A rs25648 genotyping may help identify a patient subgroup unlikely to benefit from a first-line, platinum-based combination and potential candidates for alternative therapy choices.
\end{abstract}

Original submitted 26 July 2013; Revision submitted 16 September 2013; Published online 3 October 2013

KEYWORDS: cancer chemotherapy gastric genotype polymorphisms VEGF

Although the overall incidence is constantly decreasing, gastric cancer still remains one of the leading causes of cancer death worldwide [1]. Unfortunately, in western countries more than half of gastric tumors are diagnosed in an advanced stage, when surgical resection with a curative intent is no longer possible [1]. For these patients chemotherapy is the main therapeutic option with a median overall survival (OS) rarely exceeding 10 months, response rate approaching $50 \%$ and a median progression-free survival (PFS) ranging from 5 to 7 months with the best available chemotherapy combinations $[2,3]$.

Neoplastic angiogenesis and lymphangiogenesis represent a critical process for tumor growth, invasion and metastatic spread. An altered or abnormal expression of tumor angiogenesis-related factors has also been constantly associated with a more aggressive phenotype and an increased relapse rate in several tumor types, including gastric cancer [4-10]. Interestingly, besides correlating with prognosis, tumor angiogenesis also seemed able to directly influence response/resistance to chemotherapy in clini$\mathrm{cal}$ and preclinical models [11-14]. Basic research data suggested that hypoxic conditions could impair tumor drug penetration thus limiting chemotherapy efficacy. However, factors other than drug penetrance may be equally or even more relevant in characterizing the link between tumor angiogenesis and chemotherapy efficacy.
Molecular modifications of the apoptosis regulatory pathway induced by tumor hypoxia seem to play a major role in determining radio- and chemotherapy resistance [15]. In particular, the antitumoral activity of platinum compounds proved strictly dependent on the angiogenesis conditions at the tumor site in previous observations. Clonogenic survival of colon cancer cells after oxaliplatin treatment has been shown to improve in hypoxic conditions, whereas chemotherapy-induced DNA adducts were significantly more present in aerobic tumor cells [16]. Cisplatin treatment has also been demonstrated to activate the VEGF/FLT1 autocrine-tumor signaling pathway in multiple tumor types resistant to therapy. These latter observations suggested a novel mechanism of cisplatin-induced tumor progression, where cisplatin activation of the VEGF/FLT1 pathway led to the expansion of a highly tumorigenic fraction ultimately resistant to treatment [17]. Accumulating findings suggest that the VEGF superfamily, along with their corresponding receptors, might play a crucial role in guiding the angiogenesis process in solid tumors $[4,5]$.

SNPs in the VEGF and VEGF receptor (VEGF-R) genes have been correlated with tumor neoangiogenesis through different biological mechanisms. Globally, the evidence in this area indicates that the sequence variations introduced by a given SNP might be able to

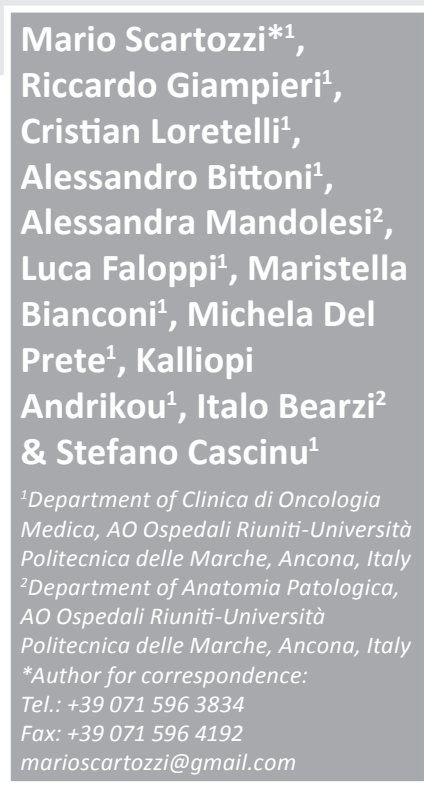

Future
Medicine $\mathrm{fS}$ 
determine a clinical effect through modification of gene-expression levels [18-23]. Exemplarily in patients with colorectal cancer, specific SNPs in the VEGF-R2 gene (also known as $K D R$ ) have in fact been shown to significantly influence microvessel density. Moreover, specific SNPs in the VEGF-A gene were shown to correlate with response to chemotherapy through modulation of tumor blood vessels [24]. In esophageal squamous carcinoma patients, the VEGF-634 C/G genotype was able to predict long-term survival after treatment with 5-fluorouracil (5-FU)/cisplatin-based chemoradiotherapy [25].

We therefore examined the role of tumor VEGF and VEGF-R polymorphisms in determining clinical outcome in metastatic gastric cancer patients receiving platinum-based firstline chemotherapy. Clinical data and SNP results for tumor VEGFs and VEGF-Rs have been analyzed with the aim of suggesting a biologically driven profile to be employed as a tool for the treatment decision process in this setting.

\section{Patients \& methods}

\section{Patient selection}

Ninety-four patients with HER-2 negative, histologically proven, metastatic gastric cancer receiving first-line platinum-based (either cisplatin or oxaliplatin combined with 5-FU) chemotherapy at our institution between January 2005 and December 2011 and whose tumor sample was available for biological analysis, were eligible for our study.

For patients who received adjuvant/neoadjuvant treatment after radical surgical resection, an interval of at least 12 months from the end of treatment was considered necessary for inclusion in the present analysis. Follow-up during chemotherapy was performed according to our institutional guidelines and consisted of physical examination, full blood count, and renal and liver function tests before each cycle. Tumor response was evaluated with CT scan of the chest and abdomen every 8 weeks by clinicians' assessment and according to the RECIST criteria (version 1.1). The site and date of progression and the date of death were recorded.

This analysis was approved by our local ethical committee.

\section{VEGF \& VEGF-R genotyping}

Genotyping was performed on formalin-fixed paraffin-embedded tissue block $(\sim 30 \mathrm{mg})$ from primary gastric tumors.

DNA was isolated from the deparaffinized tissue using the RecoverAll ${ }^{\mathrm{TM}}$ Total Nucleic
Acid Isolation Kit for FFPE Tissues (Applied Biosystems, CA, USA), according to the manufacturer's instructions. SNPs within each gene were selected using the Pupasuite software (version 2.0.0, bioinfo 2008) [101], the CIPF SNP database (dbSNP) generated by the National Center for Biotechnology Information [102] and by review of the medical literature. SNPs were selected if the polymorphism was likely to alter the gene expression in a biologically relevant manner and if it was established and welldocumented in the published literature. Globally we assumed that selected SNPs had an impact on protein expression and, as a consequence, on biological function [19].

SNP genotyping was performed using TaqMan $^{\circledR}$ technology, with SNP genotyping products (Applied Biosystems). PCR was performed and genotypes were analyzed on the 7300 RealTime PCR System (Applied Biosystems) using an ABI Prism ${ }^{\circledR} 7300$ Sequence Detection System software (version 1.3.1, Applied Biosystems). Each reaction contained $0.2 \mu$ l of total genomic DNA. Genotyping was performed by laboratory personnel blinded to patients' status. A random $10 \%$ of the samples were repeated to validate genotyping procedures.

All SNPs genotyped had to present an overall call rate of $\geq 95 \%$ to be included in our analysis.

\section{- Statistical analysis}

Statistical analysis was performed with the MedCalc software version 10.4.8 for Windows.

The association between categorical variables was estimated by $\chi^{2}$ test. Survival distribution was estimated by the Kaplan-Meier method. For statistical analysis OS and PFS were defined as the interval between the start of first-line chemotherapy to death or last follow-up visit, and as the interval between the start of first-line chemotherapy to clinical progression or death or last follow up visit if not progressed, respectively. Significant differences in probability of relapsing between the strata were evaluated by log-rank test. Hazard ratios (HRs) for median PFS and OS between groups were estimated from Cox regression models. The multivariate analysis also included adjustments for other variables such as sex (male vs female), age ( $<65$ years vs $\geq 65$ years), ECOG performance status ( $0-1$ vs $\geq 2$ ), previous gastrectomy (yes vs no), previous adjuvant chemotherapy (yes vs no), metastatic sites ( 1 vs $\geq 2$ ) and second-line chemotherapy (yes vs no). A significant level of 0.05 was chosen to assess the statistical significance. The Bonferroni correction was used to adjust multiple comparison values. 
All polymorphisms were examined for deviation from Hardy-Weinberg equilibrium using the Powermarker v. 3.25 package [103].

Linkage disequilibrium (LD) analysis was also performed using the Powermarker v. 3.25 package [103]. LD was estimated using $r^{2}$, with $r^{2}=1$ indicating complete $\mathrm{LD}$ and $\mathrm{r}^{2}=0$ indicating absent LD.

\section{Results}

\section{- Hardy-Weinberg equilibrium \& linkage disequilibrium}

The frequencies of the tested genotypes were comparable to those reported in Caucasians, with no significant deviation from the Hardy-Weinberg equilibrium. LD was not observed.

\section{Patient characteristics}

From an initial population of 124 metastatic gastric cancer patients, 94 patients $(76 \%)$ were eligible for our analysis. Main reasons for exclusion from this study were unavailability of adequate tumor samples (13 patients, 43\%), progressive disease within 12 months after the end of adjuvant/neoadjuvant treatment (seven patients, 23\%) and incomplete medical records (six patients, 20\%).

Among the study population, 65 patients were males and 29 were females with a median age at diagnosis of 65 years (range: 37-80). The extent of metastatic disease was limited to one site in as few as 23 patients $(24 \%)$, the remaining 71 patients $(76 \%)$ had two or more than two metastatic sites. Cisplatin in combination with infusional 5-FU was the preferred regimen administered (84\% of all patients), with only 15 patients (16\%) receiving an oxaliplatin-based chemotherapy combination. Forty-nine patients (52\%) received a second-line chemotherapy. In the whole group, response rate was $38 \%$, median PFS was 5.9 months and median OS was 9.1 months. All major clinical characteristics have been summarized in TabLE 1 .

\section{Univariate \& multivariate analysis results}

All SNPs analyzed showed a call rate $\geq 95 \%$. Repeated samples for genotyping validation confirmed results obtained by previous analysis in all cases.

The VEGF-A rs 25648 polymorphism was the only to correlate with response rate (TABLE 2). In fact partial remissions were four (18\%) among patients carrying the VEGF-A rs $25648 \mathrm{CT}$ or TT genotype versus 32 (44\%) among patients carrying the VEGF-A rs25648 CC genotype $(p=0.04)$. Accordingly, disease control rate (DCR; partial remission + stable disease) was obtained in nine (41\%) versus $56(77 \%)$ patients carrying with the VEGF-A rs25648 CT or TT and CC genotype (p 0.002), respectively.

The VEGF- $A$ rs2010963 and rs25648 polymorphisms, the VEGF-C rs4604600 and rs7664413 polymorphisms and the FLT4 rs307822 polymorphism, all correlated with median PFS. The VEGF-A rs25648 and the FLT4 rs307822 polymorphisms also correlated with median OS (TABle 2). Among all the other tested variables (sex, age, ECOG performance status, previous gastrectomy, previous adjuvant chemotherapy metastatic sites and second-line chemotherapy) only the use of second-line chemotherapy correlated with improved OS (10.2 months vs 6.3 months for patients who received or did not receive second-line treatment; $p=0.003)$. No other variables correlated with either response rate, PFS or OS. At multivariate analysis the use of second-line chemotherapy showed an independent role in determining median OS (HR: 0.58; 95\% CI: $0.38-0.87$ ), whereas, among all SNPs tested, only the VEGF-A rs25648 maintained an independent role in determining both median PFS (HR: 1.65; 95\% CI: 1.12-2.78; $\mathrm{p} \leq 0.0001$ ) and OS (HR: $1.58 ; 95 \%$ CI: 1.17-2.65; $\mathrm{p}=0.0003$; Figures 1 \& 2).

All major clinical characteristics were comparable between patients carrying either the $V E G F-A$ rs $25648 \mathrm{CT}$ and TT genotype or the $V E G F-A$ rs25648 C genotype (Table 1).

\section{Discussion}

The introduction of multiple chemotherapy options for first-line management of metastatic gastric cancer left the question of optimal patient selection unanswered. One of the most critical aspects limiting the full potential of these treatment choices is in fact represented by the substantial lack of clinical and biological factors able to predict the outcome of patients receiving first-line chemotherapy. In our experience the VEGF-A rs25648 CT or TT tumor genotype independently identified a subgroup of metastatic gastric cancer patients with a reduced chance of response rate, PFS and OS during firstline chemotherapy, thus indicating that tumor angiogenesis might play a central role in this setting. The relevance of tumor angiogenesis has already been suggested as a prognostic factor in gastric cancer and our findings seem to be in line with these previous observations. In fact, in a previous study in radically resected gastric 


\begin{tabular}{|c|c|c|c|c|}
\hline \multirow{2}{*}{$\begin{array}{l}\text { Patient } \\
\text { characteristics }\end{array}$} & \multirow{2}{*}{$\begin{array}{l}\text { Whole group } \\
(n=94)\end{array}$} & \multicolumn{2}{|c|}{ VEGF-A rs25648 } & \multirow[t]{2}{*}{ p-value } \\
\hline & & CT or TT $(n=22)$ & $\operatorname{CC}(n=72)$ & \\
\hline Age, years (range) & $65(37-80)$ & $64(37-78)$ & $65(38-80)$ & \\
\hline \multicolumn{5}{|l|}{ Sex, $n(\%)$} \\
\hline Males & $65(69)$ & $14(64)$ & $51(71)$ & NS \\
\hline Females & $29(31)$ & $8(36)$ & $21(29)$ & NS \\
\hline \multicolumn{5}{|l|}{ ECOG PS, $n(\%)$} \\
\hline $0-1$ & $75(80)$ & $18(82)$ & $57(79)$ & NS \\
\hline$\geq 2$ & $19(20)$ & $4(18)$ & $15(21)$ & NS \\
\hline \multicolumn{5}{|c|}{ Prior gastrectomy, $n(\%)$} \\
\hline Yes & $31(33)$ & $7(32)$ & $24(33)$ & NS \\
\hline No & $63(67)$ & $15(68)$ & $48(67)$ & NS \\
\hline \multicolumn{5}{|c|}{ Adjuvant chemotherapy, $n(\%)$} \\
\hline Yes & $18(19)$ & $3(14)$ & $15(20)$ & NS \\
\hline No & $76(81)$ & $19(86)$ & $57(80)$ & NS \\
\hline \multicolumn{5}{|c|}{ Metastatic sites, $n(\%)$} \\
\hline 1 & $23(24)$ & $5(23)$ & $18(25)$ & NS \\
\hline$\geq 2$ & $71(76)$ & $17(77)$ & $54(75)$ & NS \\
\hline \multicolumn{5}{|c|}{ First-line treatment, $n(\%)$} \\
\hline Cisplatin-5-FU & $79(84)$ & $19(86)$ & $60(83)$ & NS \\
\hline Oxaliplatin-5-FU & $15(16)$ & $3(14)$ & $12(17)$ & NS \\
\hline \multicolumn{5}{|l|}{ Response rate } \\
\hline$-P R$ & $36(38)$ & $4(18)$ & $32(44)$ & 0.04 \\
\hline$-S D$ & $29(31)$ & $5(23)$ & $24(33)$ & NS \\
\hline $\mathrm{DCR}(\mathrm{PR}+\mathrm{SD})$ & $65(69)$ & $9(41)$ & $56(77)$ & 0.002 \\
\hline$-P D$ & $29(31)$ & $13(59)$ & $16(23)$ & \\
\hline \multicolumn{5}{|c|}{ Second-line treatment, $n(\%)$} \\
\hline Yes & $49(52)$ & $12(55)$ & $37(51)$ & NS \\
\hline No & $45(48)$ & $10(45)$ & $35(49)$ & NS \\
\hline \multicolumn{5}{|l|}{ Survival (months) } \\
\hline PFS & 5.9 & 4 & 6.8 & $<0.0001$ \\
\hline OS & 9.1 & 5.9 & 9.4 & 0.0003 \\
\hline \multicolumn{5}{|c|}{$\begin{array}{l}\text { The p-values are indicated for the comparison according to VEGF-A rs } 25648 . \\
\text { 5-FU: 5-fluorouracil; DCR: Disease control rate; ECOG PS: Eastern Cooperative Oncology Group Performance Status; } \\
\text { NS: Not significant; OS: Overall survival; PD: Progressive disease; PFS: Progression-free survival; PR: Partial response; } \\
\text { SD: Stable disease. }\end{array}$} \\
\hline
\end{tabular}

cancer patients VEGF gene polymorphisms were found to be an independent prognostic marker [26].

Tissue expression of VEGF has also been correlated with prognosis in different gastric cancer series [9,10]. Furthermore VEGF gene silencing has also been demonstrated to inhibit gastric cancer growth underscoring the relevance of the VEGF driven pathway in this setting [10].

However, if on the one hand the correlation with OS may denote a prognostic role, on the other hand the effect on response rate and PFS seems to corroborate the idea of a predictive, more than a prognostic value. In this view, the possibility of an early identification of patients unlikely to benefit from a platinum-based, two drug standard regimen would potentially allow clinicians to preselect these patients for an alternative choice, thus avoiding unnecessary toxicity and hopefully improving efficacy.

We know that SNP analysis may be not easy to interpret because of the difficulty in correlating gene assay results to a critical biological effect. Accumulating evidence suggests that the interfering effect on transcriptional VEGF gene capacity and the consequent production of the corresponding VEGF protein may represent the key to interpret VEGF SNPs results. Different SNPs in the VEGF gene have in fact been associated with VEGF protein levels in many 


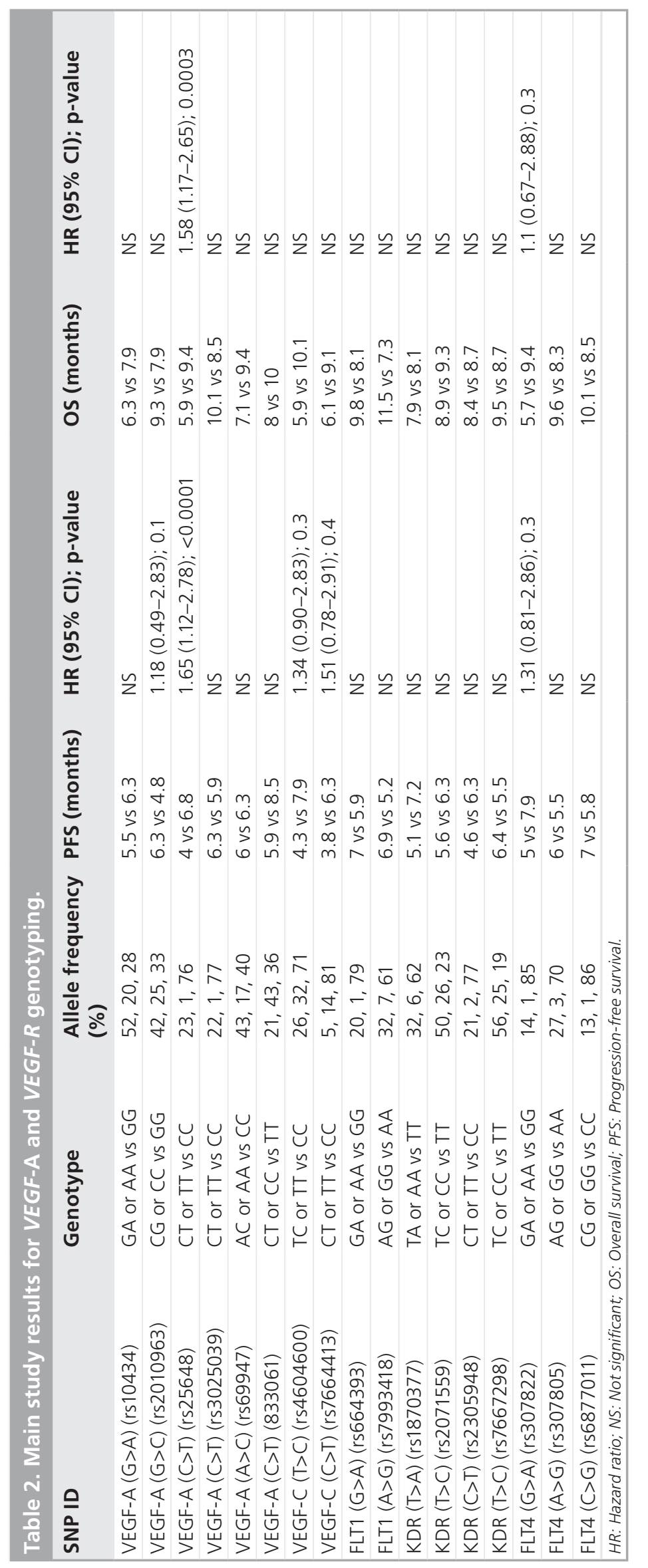




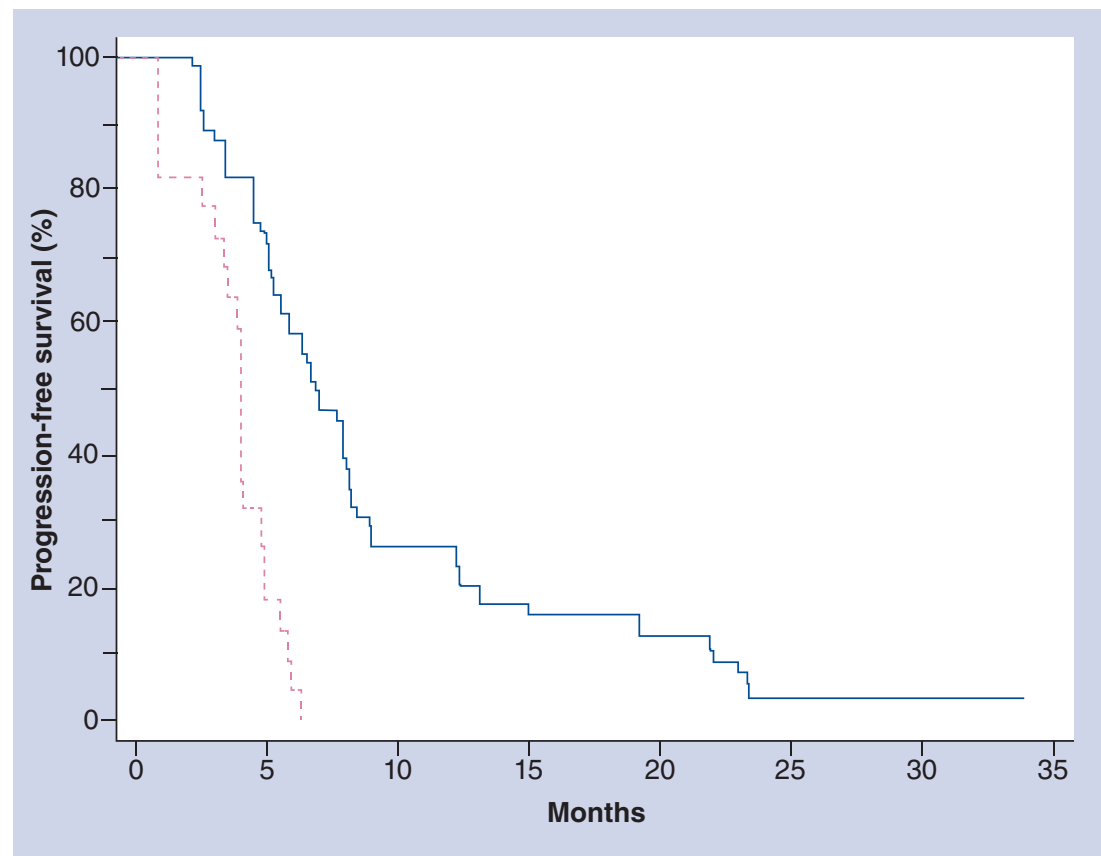

Figure 1. Kaplan-Meier curves for median progression-free survival of advanced gastric cancer patients receiving first-line, platinum-based chemotherapy showing the VEGF-A rs25648 TT or CT genotype and the VEGF-A rs25648 CC genotype. VEGF-A rs25648 TT or CT genotype (dashed line) and the VEGF-A rs25648 CC genotype (solid line; 4 months vs 6.8 months, hazard ratio: $1.65 ; 95 \% \mathrm{Cl}: 1.12-2.78 ; \mathrm{p}<0.0001)$.

tumor types. In particular $V E G F-A$ rs 25648 has been previously linked to altered serum levels of VEGF [27]. This SNP is in fact located in an

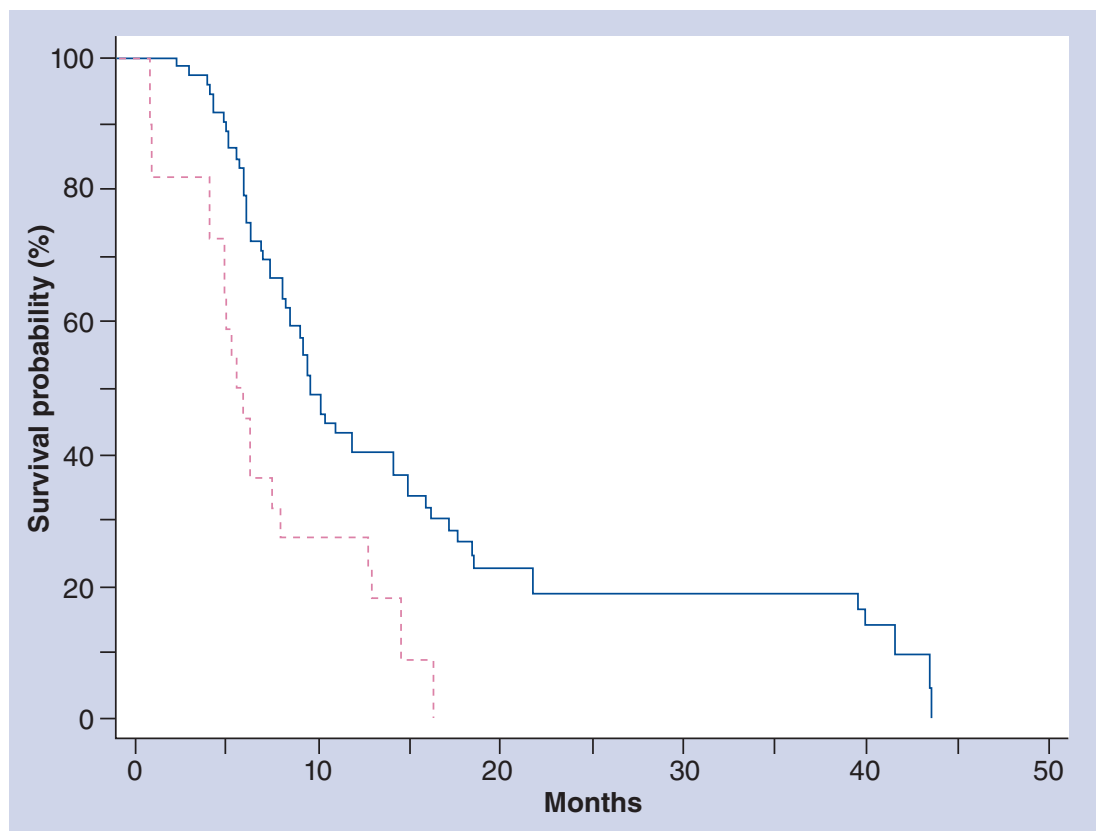

Figure 2. Kaplan-Meier curves for median overall survival of advanced gastric cancer patients receiving first-line, platinum-based chemotherapy showing the VEGF-A rs25648 TT or CT genotype and the VEGF-A rs25648 CC genotype. VEGF-A rs25648 TT or CT genotype (dashed lines) and the VEGF-A rs25648 CC genotype (solid line; 5.9 months vs 9.4 months, hazard ratio: 1.58; $95 \% \mathrm{Cl}: 1.17-2.65 ; \mathrm{p}=0.0003)$. exonic splicer enhancer site, which is known to directly influence gene transcription. We can then hypothesize that $V E G F-A$ rs 25648 polymorphic variants may be responsible for an altered gene function inducing an uncontrolled activation of the downstream signaling pathway and therefore a higher $V E G F-A$ production. A relevant role for different VEGF polymorphisms has also been previously suggested for gastric and colorectal cancer patients receiving oxaliplatin [28-30]. Although a cross-trial comparison is difficult and possibly methodologically inappropriate these findings, along with those presented in our analysis, seem to point in the direction of VEGF genotyping for patient stratification and treatment planning.

Nonetheless, before these findings can be applied in clinical practice, prospective confirmations are necessary. The relatively small sample size may in fact represent a potential limitation for a full and definitive interpretation of our results, particularly for minor frequency alleles. Moreover, in our analysis we only focused on selected angiogenesis-related genes. It is then possible that other genes not related to this pathway may also have a role in determining clinical outcome during platinumbased chemotherapy in metastatic gastric cancer patients.

\section{Future perspective}

Our observations may be crucial for the further development of both treatment and research strategy in gastric cancer. In this scenario, our data may help towards designing future clinical trials with the aim of investigating the outcome of different chemotherapy regimens in different patient groups prospectively stratified according to angiogenesis profile. Taxane-based and/or angiogenetic targeted-based therapeutic regimens may in fact represent interesting alternative options for patients deemed resistant to platinum-based combinations. Prospective trials will be essential for a full understanding and consequent clinical application of these treatment approaches.

\section{Financial \& competing interests disclosure}

The authors have no relevant affliations or financial involvement with any organization or entity with a financial interest in or financial conflict with the subject matter or materials discussed in the manuscript. This includes employment, consultancies, honoraria, stock ownership or options, expert testimony, grants or patents received or pending, or royalties.

No writing assistance was utilized in the production of this manuscript. 


\section{Ethical conduct of research}

The authors state that they have obtained appropriate institutional review board approval or have followed the principles outlined in the Declaration of Helsinki for all human or animal experimental investigations. In addition, for investigations involving human subjects, informed consent has been obtained from the participants involved.

\section{Executive summary}

\section{Background}

- Besides correlating with prognosis, tumor-driven angiogenesis also seemed able to influence response/resistance to chemotherapy in preclinical models.

\section{Patients \& methods}

- In our study we examined the correlation between angiogenesis genotyping and clinical outcome in metastatic gastric cancer patients receiving platinum-based first-line chemotherapy.

- Genotyping for VEGF-A, VEGF-C, FLT1, KDR and FLT4 was carried out on tumor samples from metastatic gastric cancer patients receiving platinum-based first-line chemotherapy.

\section{Results}

- The VEGF-A rs25648 was the only factor correlating with response rate. At multivariate analysis only the VEGF-A rs25648 maintained an independent role in determining median progression-free survival and overall survival.

\section{Discussion}

- VEGF-A rs25648 genotyping may help identify a subgroup of patients unlikely to benefit from a first-line, platinum-based combination and potential candidates for alternative therapy choices.

\section{References}

Papers of special note have been highlighted as: " of interest

" " of considerable interest

1 Jemal A, Siegel R, Ward E et al. Cancer Statistics. CA Cancer J. Clin. 60, 277-300 (2010).

2 Scartozzi M, Pistelli M, Bittoni A et al. Novel perspectives for the treatment of gastric cancer: from a global approach to a personalised strategy. Curr. Oncol. Rep. 12, 175-185 (2010).

3 Cunninhgam D, Starling N, Rao S et al. Capecitabine and oxaliplatin for advanced esophagogastric cancer. N. Engl. J. Med. 358, 36-46 (2008).

4 Yancopoule GD, Davis S, Gale NW et al. Vascular specific growth factors and blood vessel formation. Nature 407, 242-248 (2000).

5 Carmeliet P, Jain RK. Angiogenesis in cancer and other disease. Nature 407, 249-257 (2000).

6 Gluz O, Wild P, Liedtke C et al. Tumor angiogenesis as prognostic and predictive marker for chemotherapy dose-intensification efficacy in high-risk breast cancer patients within the WSG AM-01 trial. Breast Cancer Res. Treat. 126, 643-651 (2011).

7 Takahashi Y, Clearly KR, Mai M et al. Significance of vessel count and vascular endothelial growth factor and its receptor (KDR) in intestinal type gastric cancer. Clin. Cancer Res. 2, 1679-1684 (1996).

8 Kitadai Y. Angiogenesis and lymphangiogenesis of gastric cancer. J. Oncology 2010, 1-8 (2010).
9

Zhang W, He X, Ma Y et al. Inducible nitric oxide synthase expression correlates with angiogenesis, lymphangiogenesis and poor prognosis in gastric cancer patients. Hum. Pathol. 42, 1275-1282 (2011).

10 Peng L, Zhan P, Zhou Y et al. Prognostic significance of vascular endothelial growth factor immunohistochemical expression in gastric cancer: a meta-analysis. Mol. Biol. Rep. 39, 9473-9484 (2012).

11 Wang X, Chen X, Fang J, Yang C. Overexpression of both $V E G F-A$ and $V E G F-C$ in gastric cancer correlates with prognosis, and silencing of both is effective to inhibit cancer growth. Int. J. Clin. Exp. Pathol. 6, 586-597 (2013).

12 Brown JM, Wilson WR. Exploiting tumor hypoxia in cancer treatment. Nat. Rev. Cancer 4, 437-447 (2004).

- - Clear and exhaustive description of the role of tumor hypoxia in cancer treatment.

13 Brown LM, Cowen RL, Debray C et al. Reversing hypoxic cell chemoresistance in vitro using genetic and small molecules approaches targeting hypoxia inducible factor-1. Mol. Pharmacol. 69, 411-418 (2006).

14 van der Veldt AM, Lubberink M, Bahce I et al. Rapid decrease in delivery of chemotherapy to tumors after anti-VEGF therapy: implications for scheduling of antiangiogenic drugs. Cancer Cell 21, 82-91 (2012).

15 Siddiqui GK, Maclean AB, Elmasry K et al. Immunohistochemical expression of VEGF predicts response to platinum based chemotherapy in patients with epithelial ovarian cancer. Angiogenesis 14, 155-161 (2011).
16 Moeller BJ, Cao Y, Dewhirst MW. Radiation activates HIF-1 to regulate vascular radiosensitivity in tumors: role of reoxygenation, free radicals and stress granules. Cancer Cell 5, 429-441 (2004).

17 Roberts DL, Williams KJ, Cowen RL et al. Contribution of HIF-I and drug penetrance to oxaliplatin resistance in hypoxic colorectal cancer cells. Br. J. Cancer 101, 1290-1297 (2009).

18 Tsuchida R, Das B, Yeger H et al. Cisplatin treatment increases survival and expansion of a highly tumorigenic side-population fraction by upregulating VEGF/Flt1 autocrine signalling. Oncogene 27, 3923-3934 (2008).

19 Gerger A, El-Khoueiry A, Zhang W et al. Pharmacogenetic angiogenesis profiling for first-line bevacizumab plus oxaliplatin-based chemotherapy in patients with metastatic colorectal cancer. Clin. Cancer Res. 17, 5783-5792 (2011).

20 Scartozzi M, Bianconi M, Faloppi L et al. $V E G F$-And VEGFR polymorphisms affect clinical outcome in advanced renal cell carcinoma patients receiving first-line sunitinib. Br. J. Cancer 108, 1126-1132 (2013).

- Authors correlated clinical outcome and angiogenesis genotyping in renal cell carcinoma patients.

21 Hansen TF, Garm Spindler KL, Andersen RF et al. The predictive value of genetic variations in the vascular endothelial growth factor gene A in metastatic colorectal cancer. Pharmacogenomics J. 11, 53-60 (2011).

- Underscores the crucial role of VEGF-A genotyping in colorectal cancer patients receiving chemotherapy. 
22 Chen MH, Tzeng $\mathrm{CH}$, Chen PM et al. VEGF-406T>C polymorphism and its association with VEGF expression and outcome to FOLFOX-4 treatment in patients with colorectal carcinoma.

Pharmacogenomics J. 11, 227-236 (2010).

23 Formento JL, Etienne-Grimaldi MC, Francoual $\mathrm{M}$ et al. influence of the VEGF-A 936C $>$ T germinal polymorphism on tumoral VEGF expression in head and neck cancer. Pharmacogenomics 10, 1277-1283 (2009).

- Analysis that suggested a correlation between VEGF-A polymorphisms and protein expression.

24 Hansen TF, Sorensen FB, Spindler KL et al. Microvessel density and the association with single nucleotide polymorphisms of the vascular endothelial growth factor receptor 2 in patients with colorectal cancer. Virchows Arch. 456, 251-260 (2010).

25 Tamura T, Kuwahara A, Yamamori M et al. VEGF-634 C/G genotype is predictive of long-term survival after treatment with a definitive 5-fluorouracil/cisplatin-based chemoradiotherapy in Japanese patients with esophageal squamous cell carcinoma. Int. J. Med. Sci. 9, 833-837 (2012).

26 Kim JG, Sohn SK, Chae YS et al. Vascular endothelial growth factor gene polymorphisms associated with prognosis for patients with gastric cancer. Ann. Oncol. 18, 1030-1036 (2007).

27 Ruggiero D, Dalmasso C, Nutile T et al. Genetics of VEGF serum variation in human isolated populations of Cilento: importance of VEGF polymorphisms. PLoS ONE 6, e16982 (2011).

- Hypothesized a biological link between VEGF polymorphisms and VEGF serum levels.

28 Oh SY, Kwon HC, Kim SH et al. The relationship of vascular endothelial growth factor gene polymorphisms and clinical outcome in advanced gastric cancer patients treated with FOLFOX: VEGF polymorphism in gastric cancer. BMC Cancer 13, 43-50 (2013).
29 Hansen TF, Garm Spindler KL, Andersen RF et al. The predictive value of genetic variations in the vascular endothelial growth factor A gene in metastatic colorectal cancer. Pharmacogenomics J. 11, 53-60 (2011).

30 Chen MH, Tzeng CH, Chen PM et al. $V E G F-460 \mathrm{~T}-\mathrm{C}$ polymorphism and its association with VEGF expression and outcome to FOLFOX-4 treatment in patients with colorectal carcinoma.

Pharmacogenomics J. 11, 227-236 (2011).

\section{Websites}

101 Pupasuite software. http://pupasuite.bioinfo.cipf.es

102 dbSNP: Short Genetic Variations. http://www.ncbi.nlm.nih.gov/SNP

103 Powermarker v. 3.25.

http://statgen.ncsu.edu/powermarker 